\title{
CCN2: a master regulator of the genesis of bone and cartilage
}

\author{
Masaharu Takigawa
}

Published online: 22 June 2013

(C) The Author(s) 2013. This article is published with open access at Springerlink.com

\begin{abstract}
CCN family member 2 (CCN2), also known as connective tissue growth factor (CTGF), has been suggested to be an endochondral ossification genetic factor that has been termed "ecogenin", because in vitro studies revealed that CCN2 promotes the proliferation and differentiation of growth-plate chondrocytes, osteoblasts, and vascular endothelial cells, all of which play important roles in endochondral ossification. In addition to its action toward these three types of cells, CCN2 was recently found to promote the formation of osteoclasts in vitro, which cells play an important role in the replacement of cartilage by bone during endochondral ossification, thus strengthening the "ecogenin" hypothesis. For confirmation of this hypothesis, transgenic mice over-expressing $\mathrm{CCN} 2$ in cartilage were generated. The results proved the hypothesis; i.e., the over-expression of CCN2 in cartilage stimulated the proliferation and differentiation of growth-plate chondrocytes, resulting in the promotion of endochondral ossification. In addition to its "ecogenin" action, CCN2 had earlier been shown to promote the differentiation of various cartilage cells including articular cartilage cells. In accordance with these findings, cartilage-specific overexpression of $\mathrm{CCN} 2$ in the transgenic mice was shown to protect against the development of osteoarthritic changes in aging articular cartilage. Thus, CCN2 may also play a role as an anti-aging (chondroprotective) factor, stabilizing articular cartilage. CCN2 also had been shown to promote intramembranous ossification, regenerate cartilage and bone, and induce angiogenesis in vivo. For understanding of the
\end{abstract}

This manuscript is based on my presentation at the Charles Perkins Center Showcase Symposium at the University of Sydney on October 23rd 2012.

M. Takigawa $(\bowtie)$

Department of Biochemistry and Molecular Dentistry, Graduate

School of Medicine, Dentistry and Pharmaceutical Sciences,

Okayama University, 2-5-1 Shikata-cho, Kita-ku,

Okayama 700-8525, Japan

e-mail: takigawa@md.okayama-u.ac.jp molecular mechanism underlying such multifunctional actions, yeast two-hybrid analysis, protein array analysis, solid-phase binding assay, and surface plasmon resonance (SPR) analysis have been used to search for binding partners of CCN2. ECMs such as fibronectin and aggrecan, growth factors including BMPs and FGF2 and their receptors such as FGFR1 and 2 and RANK, as well as $\mathrm{CCN}$ family members themselves, were shown to bind to $\mathrm{CCN} 2$. Regarding the interaction of $\mathrm{CCN} 2$ with some of them, various binding modules in the CCN2 molecule have been identified. Therefore, the numerous biological actions of CCN2 would depend on what kinds of binding partners and what levels of them are present in the microenvironment of different types of cells, as well as on the state of differentiation of these cells. Through this mechanism, $\mathrm{CCN} 2$ would orchestrate various signaling pathways, acting as a signal conductor to promote harmonized skeletal growth and regeneration.

Keywords CCN family $\cdot \mathrm{CCN} 2 \cdot \mathrm{CTGF} \cdot \mathrm{CCN} 3 \cdot$ Chondrocytes · Osteoblasts · Vascular endothelial cells · Osteoclasts · Growth plate · Articular cartilage · Ossification · Osteoarthritis · Growth factors · Signaling · Extracellular matrix

$\begin{array}{ll}\begin{array}{l}\text { Abbreviations } \\ \text { CCN }\end{array} & \begin{array}{l}\text { Cysteine-rich 61, Connective tissue growth } \\ \text { factor, Nephroblastoma-overexpressed } \\ \text { CCN family member 2, Connective } \\ \text { tissue growth factor }\end{array} \\ \text { Hcs } 24 & \begin{array}{l}\text { Hypertrophic chondrocyte-specific } \\ \text { gene product } 24\end{array} \\ \mathrm{CCN} 3 & \begin{array}{l}\text { CCN family member 3, } \\ \text { Nephroblastoma-overexpressed }\end{array} \\ \mathrm{ECM} & \begin{array}{l}\text { Extracellular matrix } \\ \text { MMP }\end{array} \\ \text { Matrix metalloproteinase } \\ \text { BMP } & \text { Bone morphogenetic protein }\end{array}$




\begin{tabular}{|c|c|}
\hline VEGF & Vascular endothelial growth factor \\
\hline FGF & Fibroblast growth factor \\
\hline FGFR & Fibroblast growth factor receptor \\
\hline RANK & $\begin{array}{l}\text { Receptor activator of nuclear factor } \\
\text { kappa-B }\end{array}$ \\
\hline RANKL & $\begin{array}{l}\text { Receptor activator of nuclear factor } \\
\text { kappa-B ligand }\end{array}$ \\
\hline OPG & Osteoprotegerin \\
\hline SPR & Surface plasmon resonance \\
\hline IGFBP & Insulin-like growth factor-binding protein \\
\hline IGF & Insulin-like growth factor \\
\hline IGFR & Insulin-like growth factor receptor \\
\hline VWC & von Willebrand factor type $\mathrm{C}$ repeat \\
\hline TSP & Thrombospondin \\
\hline $\mathrm{FN}$ & Fibronectin \\
\hline DC-STAMP & Dendric cell-specific transmembrane protein \\
\hline TrkA & Tropomyosin-related kinase A \\
\hline$N F-k B$ & Nuclear factor-kappa B \\
\hline
\end{tabular}

\section{Introduction}

Bone is formed by two processes, intramembranous ossification and endochondral ossification (Fig. 1). In the process of intramembranous ossificiation, osteoblasts differentiated from mesenchymal stem cells directly form bone. On the other hand, in the process of endochondral ossification, chondrocytes differentiated from mesenchymal stem cells first form transient cartilage, the so-called growth-plate cartilage, and then this cartilage is replaced by bone after invasion of blood vessels into the cartilage. Even after that, articular cartilage remains as a permanent cartilage on the surface of joints.

As shown in Fig. 2, in the fetus, bone anlagen is formed as cartilage; and then intramembranous ossification occurs at the

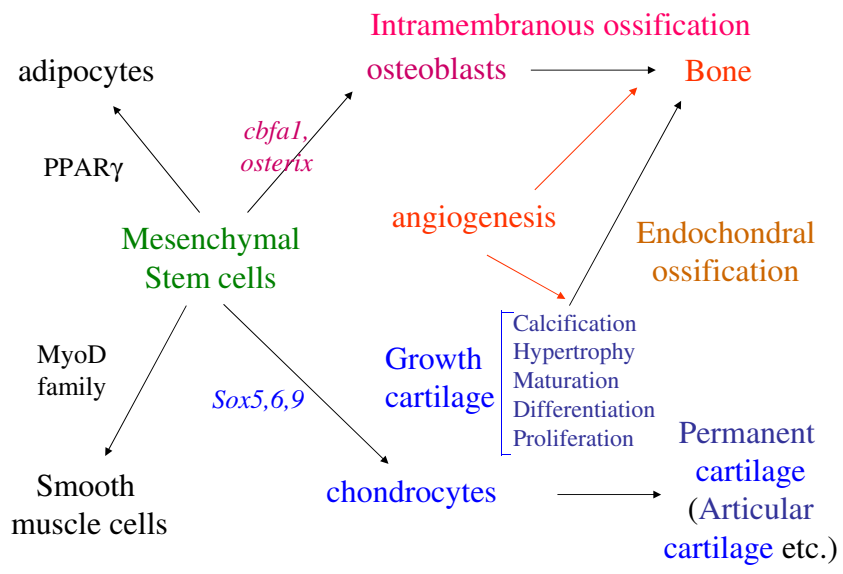

Fig. 1 Mechanism of skeletal formation. Bone is formed by two processes, intramembranous ossification and endochondral ossification. Cartilage is classified into two categories, permanent cartilage such as articular cartilage and temporal cartilage, which is involved in endochondral ossification center of this anlagen, referred to as the primary ossification center. Then the bone expands in both directions, and a secondary ossification center is formed in the epiphysis. As a result, plate-like cartilage remains between both bones; and this plate-like cartilage is called the growth plate.

In the growth plate, chondrocytes proliferate, differentiate, mature, and become hypertrophic chondrocytes which form a calcified matrix. Then, osteoclasts erode this calcified cartilage, after which blood vessels penetrate into the eroded cartilage. Also, osteoblasts deposit a bone matrix; and finally the cartilage is replaced by bone. Many growth factors, cytokines, and vitamins have been shown to be involved in chondrocyte proliferation and differentiation, but none of them promotes the entire process of endochondral ossification. To isolate such a factor, we previously carried out differentialdisplay PCR and cloned a gene that was predominantly expressed in hypertrophic chondrocytes. We named it hypertrophic chondrocyte-specific gene hes 24, the product of which was found to be identical to CTGF, now called CCN2 (Fig. 2; Takigawa 2003; Takigawa et al. 2003; Perbal and Takigawa 2005; Nakanishi et al. 1997).

$\mathrm{CCN} 2$ is a member of the CCN protein family, which originally consisted of 3 members, i.e., CTGF, Cyr61 and Nov, and now consists of 6 members (Takigawa et al. 2003; Perbal and Takigawa 2005; Brigstock et al. 2003). These members are cysteine-rich secretory proteins, and each member contains four conserved modules, i.e., an IGFBP module, VWC type $\mathrm{C}$ module, TSP type 1 repeat, and C-terminal module except for CCN5, which lacks the CT module (Fig. 3, top). Early studies paid much attention to the involvement of CCN2 in fibrosis, because expression of the CCN2 gene had been shown to be induced by TGF-beta, which expression had been found in various fibrotic disorders, and because CCN2 had been shown to promote the proliferation, migration, and adhesion of fibroblasts (Perbal and Takigawa 2005; Takigawa 2003; Mori et al. 1999; Sato et al. 2000; Brigstock 1999; Moussad and Brigstock 2000). However, the discovery of $\mathrm{CCN} 2$ in chondrocytes opened a new era for $\mathrm{CCN} 2$ research, especially that regarding its physiological function.

\section{Role of CCN2 in endochondral ossification - in vitro studies}

By making recombinant proteins, my colleagues and I found that $\mathrm{CCN} 2$ stimulates the proliferation and differentiation of growth-plate chondrocytes and causes them to become hypertrophic and form a calcified matrix (Nakanishi et al. 2000). We also found that recombinant $\mathrm{CCN} 2$ ( $\mathrm{rCCN} 2)$ promotes the proliferation and differentiation of osteoblasts, as well as calcification by them (Nishida et al. 2000). Moreover, we found $\mathrm{CCN} 2$ to promote the proliferation, adhesion, migration, and tube formation of vascular endothelial cells and to 
Fig. 2 (Right) Process of endochondral ossification. Long bones are first formed as cartilage template and then ossification occurs at the center of the cartilaginous bone anlagen, which is called the "primary ossification center." After a secondary ossification center is formed, the cartilage between primary and secondary ossifications, which is called the growth plate, grows rapidly and is then replaced by bone after invasion of blood vessels into the cartilage. (Left) $\mathrm{CCN} 2$ gene was cloned as a hypertrophic chondrocyte specific gene 24 (hcs24). Its gene product promotes proliferation and differentiation of chondrocytes, osteoblasts, and vascular endothelial cells

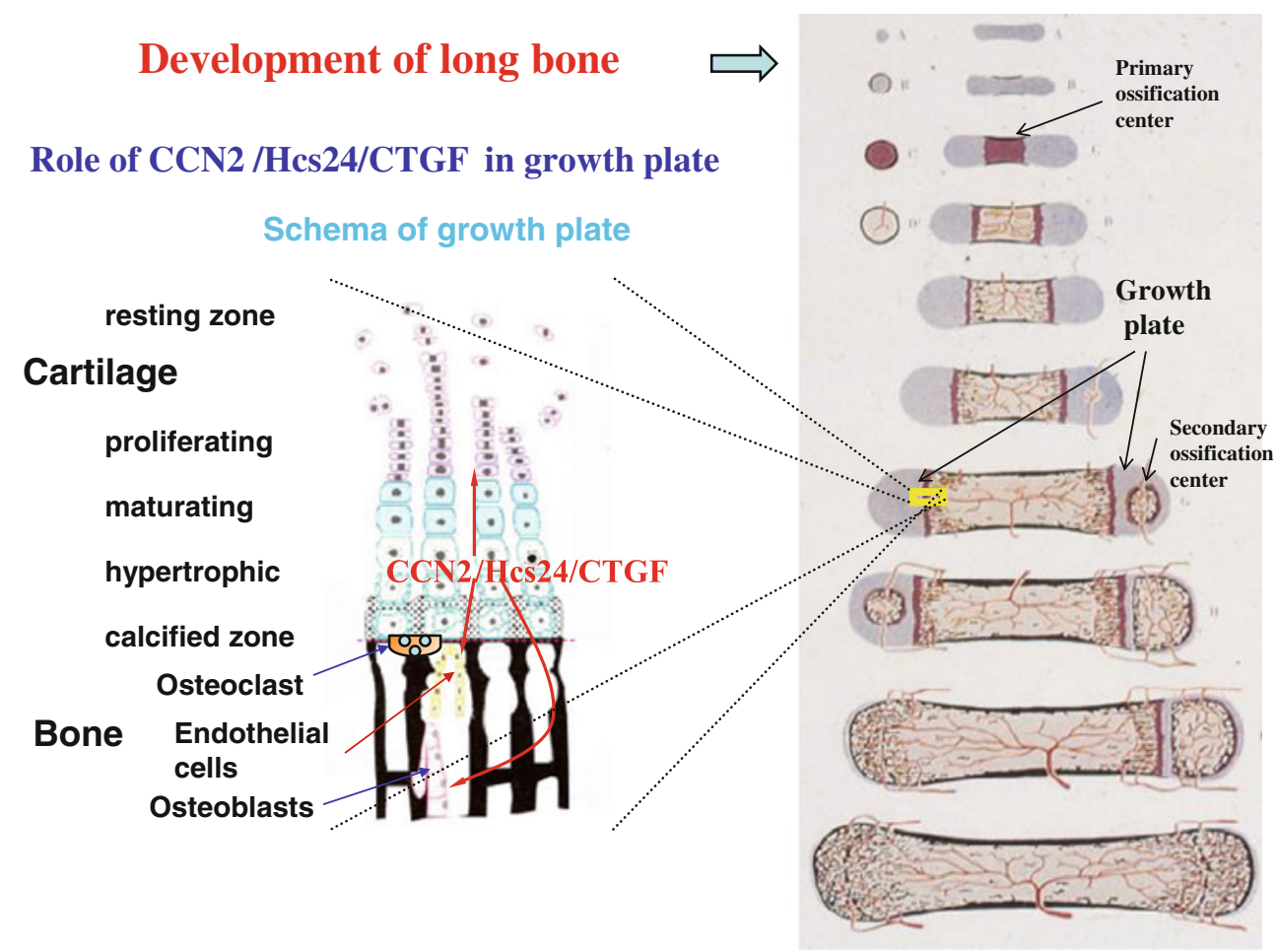

induce angiogenesis in ovo and in vivo (Shimo et al. 1998, 1999; Kubota and Takigawa 2007a).

From these findings, we hypothesized that $\mathrm{CCN} 2$ promotes endochondral ossification by acting on three types of cells: chondrocytes, osteoblasts, and endothelial cells (Takigawa et al. 2003; Kubota and Takigawa 2007b, 2011). Thus we coined the term "Ecogenin: endochondral ossification genetic factor" to describe this molecule (Takigawa et al. 2003). However, there is one more type of cell that plays an important role in endochondral ossification, i.e., the osteoclast. As shown in
Fig. 2, when cartilage is replaced by bone, osteoclasts invade into the matrix of the calcified cartilage and provide space for the deposition of osteoid by osteoblasts. Therefore, we recently investigated the effect of CCN2 on osteoclastogenesis.

Using osteoclast precursor cell line RAW 264.7 cells (Shui et al. 2002), we found that (1) CCN2 potentiates RANKL-induced osteoclastogenesis in its late stage; (2) expression of CCN2 and DC-STAMP is induced in the late stage of RANKL-induced osteoclastogenesis, with the induction of CCN2 being earlier than that of DC-STAMP; and
Fig. 3 (Top) Modular structure of CCN2. CCN2 consists of the following four conserved modules: insulin-like growth factor binding protein-like (IGFBP) module, Willebrand factor type C (VWC) module, thrombospondin (TSP) type 1 repeat, and $\mathrm{C}$-terminal module. (middle and bottom) Binding partners of $\mathrm{CCN} 2$ protein modules and function of each module. Other $\mathrm{CCN}$ family proteins in parentheses show that the molecules indicated are their binding partners. FGFR1 binds to CCN2 through other modules except CT. The molecules marked in red were found to bind to CCN2 in the author's laboratory

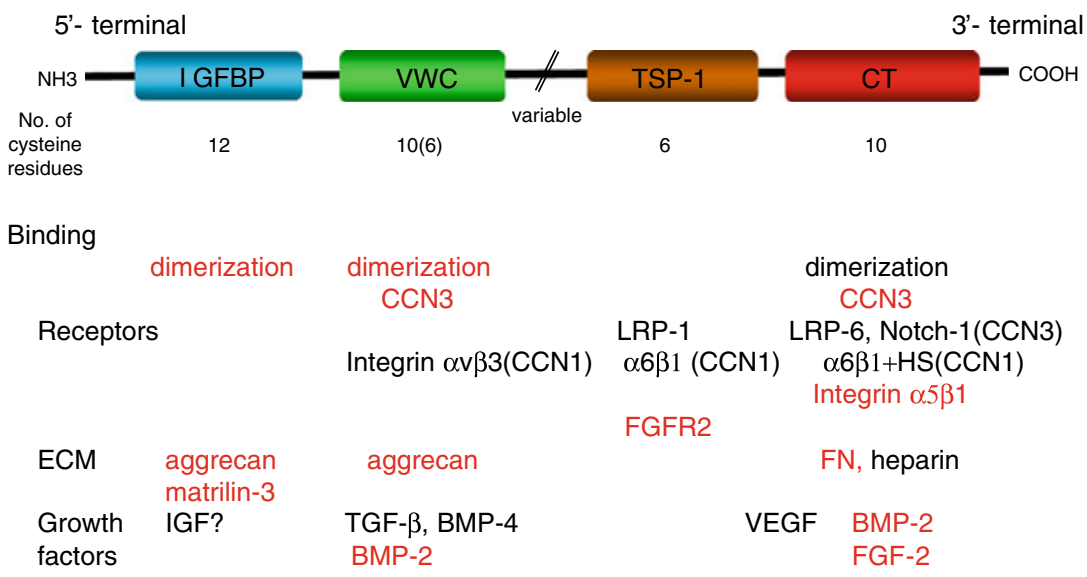

binding module unidentified molecules: $280 \mathrm{kDa}$ chondrocyte surface protein, TrkA, RANK, OPG, FGFR1[except for CT], DC-STAMP, perlecan, decorin, MMPs

cell proliferation cell adhesion 
(3) CCN2 potentiates the expression of DC-STAMP in the late stage of osteoclastogenesis (Nishida et al. 2011a; Fig. 4). Moreover, using IP-Western blotting and the solidphase binding assay, we also found that $\mathrm{CCN} 2$ binds to DCSTAMP (Nishida et al. 2011a; Fig. 4).

Using fetal liver cells as a source of osteoclast precursor from CCN2 null mice (Nishida et al. 2011a), we also found that osteoclastogenesis induced by M-CSF and RANKL in vitro is impaired in these mice and that exogenous CCN2 rescues this impaired osteoclastogenesis. Furthermore, we showed that knock down of CCN2 by siRNA inhibits pit formation, which is a marker of active osteoclasts, and that this inhibition is reversed by the addition of rCCN2 (Nishida et al. 2011a). Moreover, CCN2 increases DC-STAMP expression in fetal liver cells from CCN2 null mice; and forced expression of DC-STAMP by retrovirus transfer increases the formation of TRAP-positive cells from fetal liver cells (Nishida et al. 2011a). These findings indicate that CCN2 induces the late stage of osteoclastogenesis through DCSTAMP. In summary, as shown in the right part of Fig. 4., RANKL stimulates osteoclast precursors to form Trappositive cells, which then produce $\mathrm{CCN} 2$; and this $\mathrm{CCN} 2$ induces the expression of DC-STAMP and interacts with the DC-STAMP to promote the fusion of the TRAP-positive cells, thus resulting in enhancement of late-stage osteoclastogenesis.

Moreover, CCN2 enhances the early stage of osteoclastogenesis (Aoyama et al. 2013). As shown in the left part of Fig. 4, we revealed by performing the solid-phase binding assay and using surface plasmon resonance (SPR) that CCN2 binds to RANK. SPR revealed that CCN2 binds to RANK with a KD value of less than $100 \mathrm{nM}$ (Aoyama et al. 2013). Interestingly, CCN2 also binds to osteoprotegerin (OPG), which is a decoy receptor of RANK (Aoyama et al. 2013). Translocation of $\mathrm{NF}-\mathrm{KB}$ into the nucleus is known to be one of the signaling pathways of RANKL (Hu et al. 2008); and CCN2 enhances this
RANKL-induced nuclear translocation of NF-KB in RAW264.7 cells, which are osteoclast precursor cells (Aoyama et al. 2013). In addition, CCN2 enhances RANKL-induced phosphorylation of JNK, ERK, and p38 in RAW264.7 cells (Aoyama et al. 2013). Therefore, these findings strongly suggest that in the early stage CCN2 interacts with RANK and enhances RANKL signaling, leading to osteoclastogenesis (Aoyama et al. 2013). This effect may be due to increased availability of RANKL as a result of the binding of $\mathrm{CCN} 2$ to OPG. However, since we also found that OPG inhibits the interaction between CCN2 and RANK, OPG might inhibit the enhancement of RANKL signaling by CCN2 (Aoyama et al. 2013).

In conclusion, $\mathrm{CCN} 2$ also stimulates osteoclastogenesis in addition to acting on chondrocytes, osteoblasts, and endothelial cells. These findings strongly support our hypothesis that CCN2 is an endochondral ossification genetic factor ("ecogenin," Fig. 5). However, this hypothesis comes from the data obtained mainly from in vitro experiments (Fig. 5). Therefore, we needed to confirm this hypothesis by performing in vivo studies, the results of which are described in the next section.

\section{Generation of transgenic mice overexpressing CCN2 in cartilage proves the "ecogenin" action of $\mathrm{CCN} 2$}

To confirm that CCN2 actually promotes endochondral ossification in vivo, we generated transgenic mice over-expressing a ccn $2 /$ lac $Z$ fusion gene in cartilage under the control of the $6 \mathrm{~kb}$ Col2al-enhancer/promoter (Tomita et al. 2013). Although there was no difference between WT and TG mice on embryonic day 15.5, the TG mice on postnatal day 1 were larger than the WT ones. Also at 8 weeks, these TG mice were larger than the WT animals (Tomita et al. 2013). When we measured the length of their long bones, the tibial length was longer in the TG mice than in the WT ones on postnatal day 1. Statistical analysis
Fig. 4 Mechanism of CCN2enhanced osteoclastogenesis. (Right) CCN2 expressed during osteoclastogenesis promotes osteoclast formation via induction of and interaction with DC-STAMP in the late stage of osteoclastogenesis. In the early stage of osteoclastogenesis, CCN2 interacts with RANK and enhances RANKL signaling, probably due to increased availability of RANKL, because $\mathrm{CCN} 2$ also interacts with OPG, a decoy receptor of RANK. The figure was drawn with help by Drs. T. Nishada and E. Aoyama

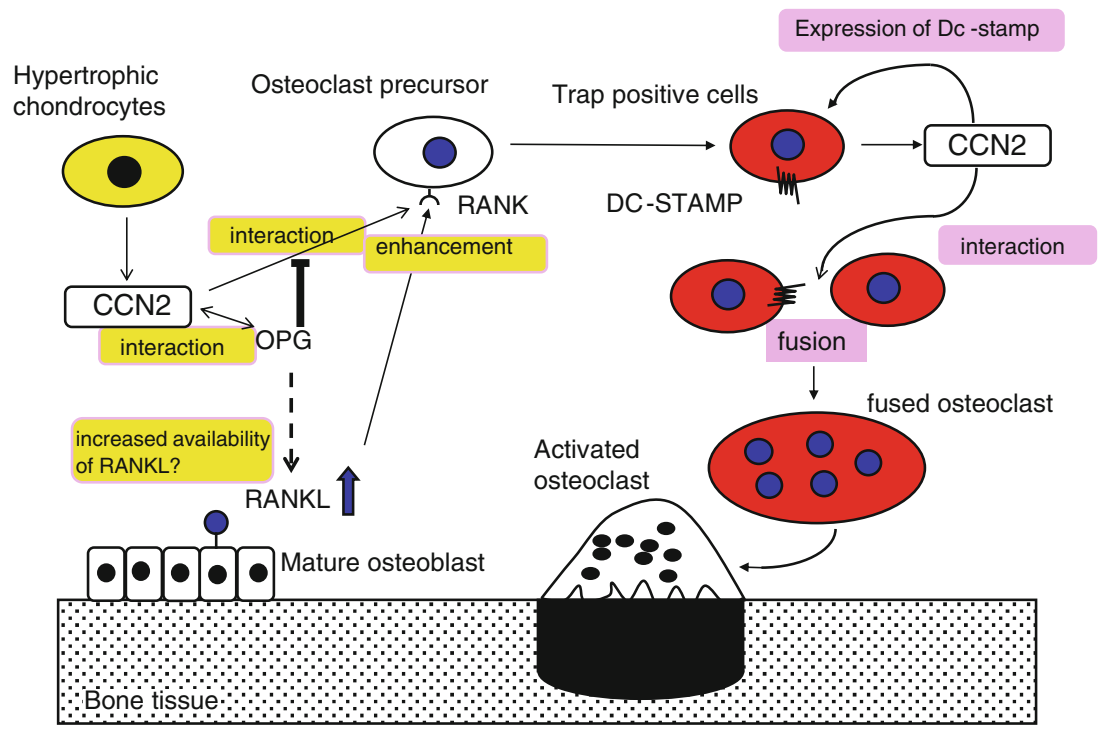


Fig. 5 CCN2 enhances osteoclastogenesis in addition to promoting actions toward chondrocytes, osteoblasts, and vascular endothelial cells. In other words, CCN2 promotes endochondral ossification by acting on all four types of cells that are involved in endochondral ossification, thus providing the so-called "Ecogenin" (Endochondral ossification genetic factor) action of CCN2. Modified from Takigawa et al. (2003)

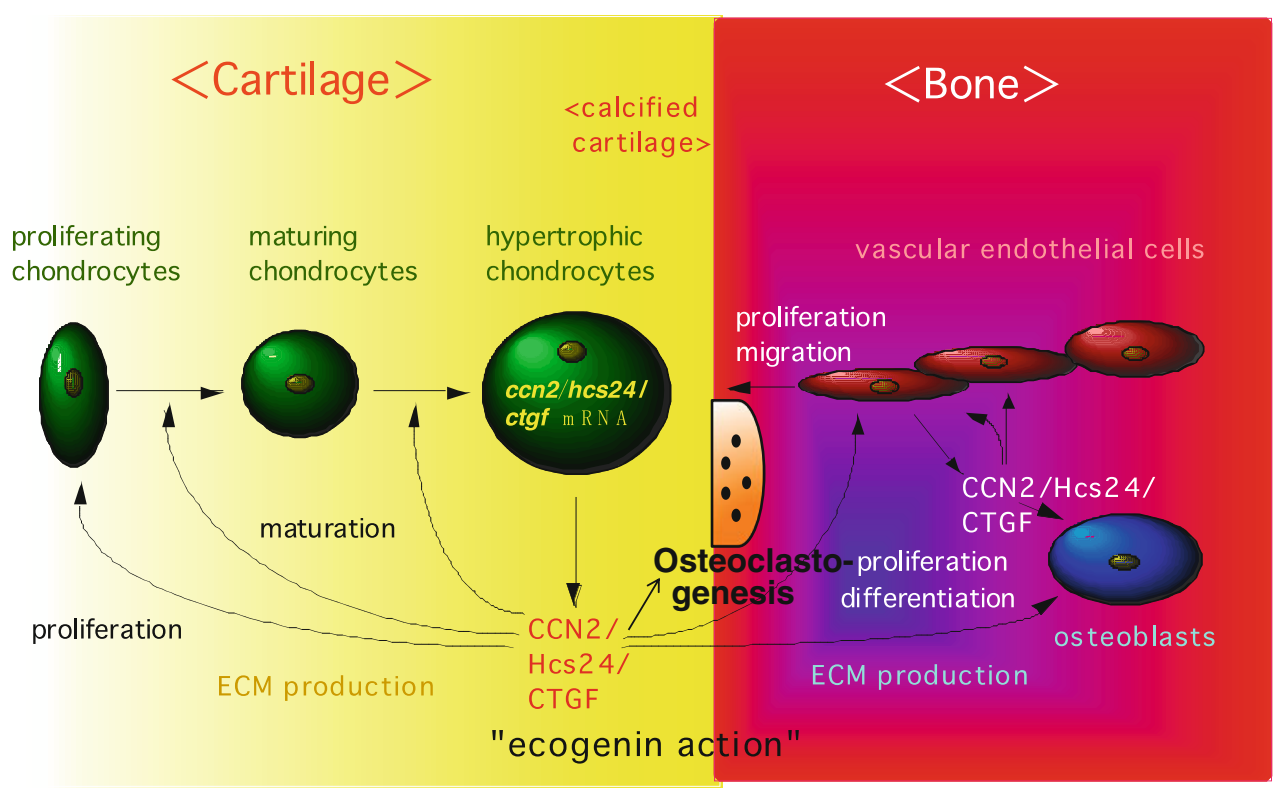

using P2 mice also confirmed that the TG bone was longer than the WT bone. Newborn transgenic mice also showed extended length of their long bones (Tomita et al. 2013). In order to assess whether the enhanced bone growth of CCN2 transgenic animals was due to enhanced cell proliferation, we stained sections of E19.5-day transgenic and wt embryos with an antibody against proliferative cell nuclear antigen (PCNA). The data showed that over-expression of CCN2 stimulated chondrocyte proliferation predominantly in the proliferative zone, but also in the resting zone (Tomita et al. 2013). Histological and immunohistological analyses revealed increased accumulation of proteoglycans and collagen II in the $\mathrm{Tg}$ mice (Tomita et al. 2013). The results of a TUNEL assay revealed marginally enhanced accumulation of apoptotic cells at the cartilage-bone interface and in the adjacent subchondral zone in the transgenic embryos as compared with that in the wildtype mice (Tomita et al. 2013). In in vitro short-term cultures of chondrocytes prepared from the cartilage of ccn2-over-expressing mice, the expression of col2al, aggrecan and ccn2 was substantially enhanced; and in long-term cultures the expression levels of these genes were further enhanced (Tomita et al. 2013). The expression of Colloa1, a marker of hypertrophy, and that of vegf and of $m m p-9$, both vascular invasion factors expressed in the hypertrophic zone and boundary between cartilage and bone, were also enhanced; however, the level of stimulation in the transgenic chondrocytes was not as high as that for aggrecan or Col2al (Tomita et al. 2013). In addition, in vitro chondrogenesis by rib chondroblasts from ccn2-over-expressing mice was strongly enhanced (Tomita et al. 2013). $I G F-I$ and $I G F-I I$ mRNA levels were elevated in the transgenic chondrocytes, and treatment of non-transgenic chondrocytes with $\mathrm{CCN} 2$ stimulated the expression of these mRNAs (Tomita et al. 2013). The addition of CCN2 induced phosphorylation of IGFR, and ccn2-overexpressing chondrocytes showed enhanced phosphorylation of IGFR, suggesting that our observation made on TG cartilage may have been mediated in part by $\mathrm{CCN} 2$-induced overexpression of IGF-I and IGF-II (Tomita et al. 2013). Micro-CT analysis revealed that overexpression of CCN2 increased bone density, the extent of mineralization of cancellous bone, and the thickness of the cortical bone (Tomita et al. 2013).

These findings indicate that overexpression of CCN2 in transgenic mice accelerated the endochondral ossification processes by promoting the proliferation and differentiation of growth-plate chondrocytes, resulting in increased length of their long bones (Tomita et al. 2013; Fig. 6). These results also indicate the possible involvement of locally enhanced IGF-I or IGF-II in this extended bone growth (Tomita et al. 2013).

\section{Chondroprotective action of CCN2 in articular cartilage shown by the transgenic mice overexpressing CCN2 in cartilage}

CCN2 stimulates the synthesis of DNA and proteoglycans in articular chondrocytes as in growth-plate chondrocytes, but unlike its stimulation of calcification by growth cartilage cells, CCN2 does not stimulate undesired calcification in articular chondrocytes (Nishida et al. 2002; Kubota and Takigawa $2007 b, 2011)$. Also, the administration of CCN2 together with gelatin hydrogel into cartilage defects repairs articular cartilage (Nishida et al. 2004; Kubota and Takigawa 2007b, 2011). These facts let us to speculate some special effect of CCN2 on the articular cartilage in these CCN2-overexpressing mice described above.

To investigate the effect of CCN2 overexpression on articular cartilage (Itoh et al. 2009, 2011), we used a spontaneously 
Fig. 6 Overexpression of $\mathrm{CCN} 2$ in the cartilage of transgenic mice promotes proliferation, differentiation, and hypertrophy of chondrocyes, resulting in elongation of the long bones. In other words, this in vivo experiment confirmed that $\mathrm{CCN} 2$ promotes endochondral ossification. The figure was drawn with help by Drs. N. Tomita and T. Hattori

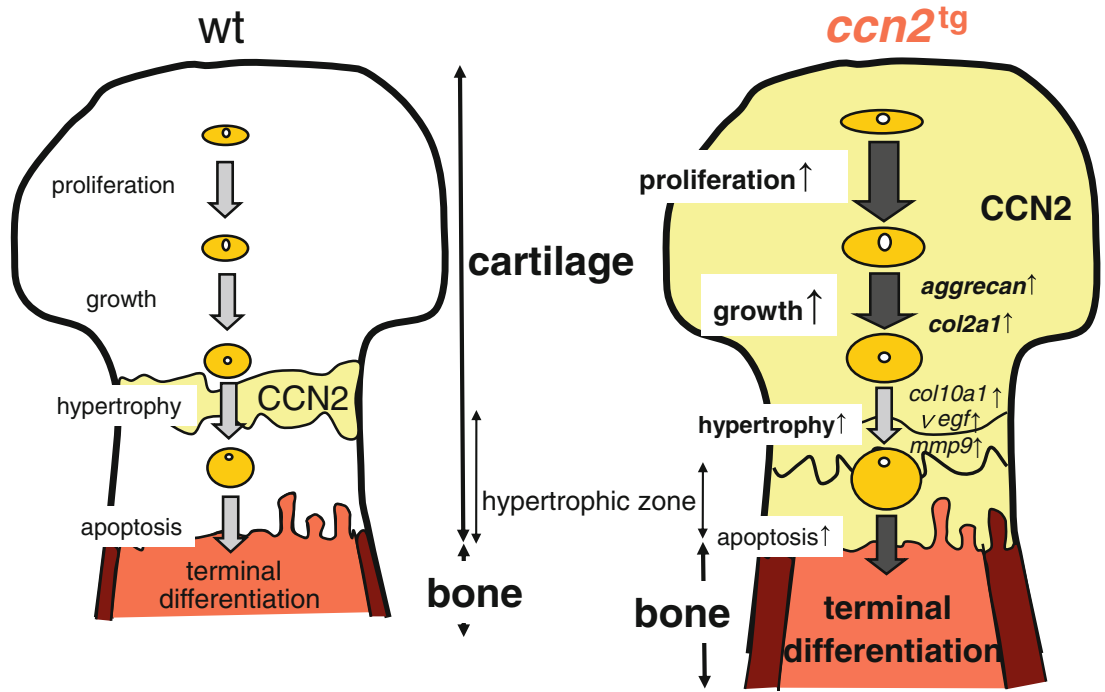

occurring osteoarthritis mouse model. We kept the WT and the TG mice for approximately 20 months and then carried out radiographic analysis of their knee joints. The results indicated that $50 \%$ of the WT mice showed an osteoarthritis-like phenotype in their knee joints, as indicated by a narrowed joint space and rough cartilage surface (Itoh et al. 2009, 2011). On the other hand, no TG mice showed such an age-related osteoarthritis-like change (Itoh et al. 2009, 2011).

To confirm the enhanced expression of the $c c n 2$ transgene in the articular cartilage in these old mice, we performed immunohistochemical analysis with ant-CCN2 antibody and found enhanced accumulation of CCN2 in the superficial and deep zones of the articular cartilage of knee joints from 21month-old TG mice (Itoh et al. 2009, 2011). Also, histological analysis showed that TB staining, which is a marker of proteoglycan accumulation, was more intense in the TG mice than in the WT ones (Itoh et al. 2009, 2011). Moreover, immunostaining with anti-type II collagen showed that the collagen content did not decrease during aging in the TG mice, whereas it did decrease in the WT mice (Itoh et al. 2009, 2011). It is well known that collagen type I and X and MMP-13 are not detectable in normal articular cartilage and that the appearance of these proteins in articular cartilage is indicative of osteoarthritis (Kawaguchi 2008; Aigner et al. 1993; Shlopov et al. 2000). Therefore, we investigated the presence of these proteins in articular cartilage of these mice by immunostaining. As a result, age-related osteoarthritic changes such as the production of collagen types I and X and MMP-13 were not detected in the articular cartilage of the CCN2-overexpressing mice, in contrast to the positive staining for these three proteins in the WT articular cartilage (Itoh et al. 2009, 2011). In conclusion, cartilage-specific overexpression of $\mathrm{CCN} 2$ protected against the development of osteoarthritic changes in aging articular cartilage (Itoh et al. 2009, 2011). Thus, CCN2 may play a role as an anti-aging (chondroprotective) factor, stabilizing articular cartilage.

\section{Molecular mechanism of CCN2-induced harmonized skeletal growth and regeneration}

As was shown in Fig. 2, in the case of growth cartilage cells, CCN2 promotes not only proliferation and cartilage matrix formation, but also hypertrophy and calcification (Nakanishi et al. 2000). The latter are characteristics of growth cartilage cells. However, in the case of articular cartilage cells, CCN2 does not cause hypertrophy or calcification, which are undesired and pathological characteristics for articular cartilage (Nishida et al. 2002). Moreover, in the case of auricular chondrocytes, CCN2 promotes their proliferation and formation of cartilage matrix, which includes elastin, a major marker of elastic cartilage; but CCN2 does not cause hypertrophy of or calcification by these auricular chondrocytes (Fujisawa et al. 2008). Moreover, in vivo administration of CCN2 with gelatin hydrogel into a defect in articular cartilage regenerates the cartilage without undesired calcification (Nishida et al. 2004). Furthermore, CCN2 stimulates the expression of the differentiated phenotype of different types of cells such as osteoblasts (Nishida et al. 2000; Takigawa et al. 2003; Kawaki et al. 2008b, 2011), vascular endothelial cells (Shimo et al. 1998, 1999; Takigawa et al. 2003), and osteoclasts (Nishida et al. 2011a). CCN2 is also expressed in osteocytes (Kawaki et al. 2011) in developing bone; and mechanical stress, which is important for bone homeostasis, induces CCN2 expression in osteocytes (Yamashiro et al. 2001). $\mathrm{CCN} 2$ is also required for intramembranous ossification (Kawaki et al. 2008b), and in vivo adiministration of CCN2 with gelatin hydrogel into the defect of bone regenerates bone through intramembrabous ossification (Kikuchi et al. 2008). Moreover, CCN2 is expressed in sites of rat alveolar bone regeneration after tooth extraction (Kanyama et al. 2003) and during distraction osteogenesis (Kadota et al. 2004), suggesting that $\mathrm{CCN} 2$ plays an important role in regeneration. In other 
words, CCN2 promotes harmonized skeletal growth and maintenance.

How does CCN2 exhibit such a harmonizing action? What is the molecular mechanism of its action? CCN proteins have four modules, and many investigators including us have reported that each module has various binding partners, as described below. This might be a major reason for its harmonizing action (Fig. 3).

\section{Extracellular matrices (ECMs) and integrins}

Using ${ }^{125}$ I-labled CCN2, we previously revealed that CCN2 binds to perlecan, a heparan sulfate proteoglycan (Fig. 3, Nishida et al. 2003); but at that time we could not identify which domain was involved in this binding. Searching for CCN2-interactive proteins by yeast-two-hybrid screening, we identified the fibronectin 1 gene product as a major binding partner of $\mathrm{CCN} 2$ in the chondrocytic cell line HCS-2/8 (Fig. 3, Hoshijima et al. 2006). Only the CT domain of CCN2 bound directly to fibronectin (FN). CCN2 and its CT domain enhanced the adhesion of HCS$2 / 8$ cells to FN. The CCN2-enhancing effect on cell adhesion to FN was abolished by anti-alpha5betal integrin (alpha5beta1), suggesting that $\mathrm{CCN} 2$ enhanced chondrocyte adhesion to $\mathrm{FN}$ through direct interaction of its C-terminal CT domain with FN and that integrin alpha5betal was involved in this adhesion (Hoshijima et al. 2006).

The yeast-two-hybrid screening also revealed that CCN2 binds to aggrecan and that its binding is through IGFBP and VWC modules (Fig. 3, Aoyama et al. 2009). SPR (surface plasmon resonance) analysis showed the direct interaction between $\mathrm{CCN} 2$ and aggrecan, and ectopically overexpressed $\mathrm{CCN} 2$ and G3 domain of aggrecan core protein confirmed their binding in vivo. When IGFBP-VWC modules, but not TSP-CT modules, were expressed in chondrocytic HCS-2/8 cells, aggrecan production in the cells was strongly induced, suggesting that this binding may be related to the $\mathrm{CCN} 2$-enhanced production and secretion of aggrecan by chondrocytes.

Moreover, $\mathrm{CCN} 2$ has been shown to bind to various ECMs such as heparan sulfate proteoglycan via its CT module (Nishida et al. 2003; Gao and Brigstock 2004), matrilin-3 via IGFBP-VWC modules (Araki et al. 2008), and decorin, in which case the binding module in the CCN2 molecule has not been identified (Vial et al. 2011; Fig. 3). CCN2 is also known to bind ECM-degrading enzymes such as matrix metalloproteinases (MMPs; Fig. 3), which cleave CCN2 (Hashimoto et al. 2002).

In addition to binding to integrin alpha5beta1, CCN2 and other $\mathrm{CCN}$ family members are reported to bind to various other integrins that are receptors for ECMs (for reviews, see Jun and Lau 2011; Lau and Lam 1999, and Kubota and Takigawa 2007b)
Growth factors and their receptors

Because it had been reported that CCN2 can antagonize BMP-4 activity by preventing its binding to BMP receptors in Xenopus embryos (Fig. 3, Abreu et al. 2002) and because both CCN2 (Kubota and Takigawa 2007b) and BMP-2 (Yoon and Lyons 2004) play an important role in cartilage metabolism, we first evaluated whether or not $\mathrm{CCN} 2$ could interact with BMP-2; and so we examined the combination effect of CCN2 with BMP-2 (CCN2-BMP-2) on the proliferation and differentiation of chondrocytes (Maeda et al. 2009). Immunoprecipitation-Western blotting analysis and a solid-phase binding assay revealed that $\mathrm{CCN} 2$ directly interacted with BMP-2, probably via its CT module and IGFBP-VWC modules (Fig. 3), and surface plasmon resonance (SPR) spectroscopy showed that its dissociation constant was $0.77 \mathrm{nM}$ (Maeda et al. 2009). CCN2 was colocalized with BMP-2 at the pre-hypertrophic region in the E18.5 mouse growth plate (Maeda et al. 2009). Interestingly, CCN2-BMP-2 did not affect the BMP-2/CCN2-induced phosphorylation of $\mathrm{p} 38 \mathrm{MAPK}$, but caused less phosphorylation of ERK1/2 in cultured chondrocytes (Maeda et al. 2009). CCN2-BMP-2 stimulated cell growth to a lesser degree than that achieved by either CCN2 or BMP-2 alone; whereas the expression of chondrocyte marker genes and proteoglycan synthesis, representing the mature chondrocytic phenotype, was increased collaboratively by CCN2-BMP-2 treatment in the cultured chondrocytes (Maeda et al. 2009). Because phosphorylation of ERK1/2 by $\mathrm{CCN} 2$ is involved in chondrocyte proliferation (Yosimichi et al. 2001, 2006), CCN2-BMP-2 treatment might promote chondrocyte differentiation by suppressing chondrocyte proliferation via decreased ERK1/2 phosphorylation. In any event, the above findings suggest that CCN2 may regulate the proliferating and differentiation of chondrocytes by forming a complex with BMP-2 as a novel modulator of BMP signaling (Maeda et al. 2009).

In addition to BMP signaling, FGF signaling is important in the developing endochondral skeleton (Ornits 2005). To investigate whether $\mathrm{CCN} 2$ interacts with fibroblast growth factor 2 (FGF2), we performed a solid-phase binding assay and immunoprecipitation-Western blot analysis and found that the C-terminal module of CCN2 (CT) directly bound to FGF2 (Fig. 3, Nishida et al. 2011b). SPR spectroscopy revealed that the dissociation constant between CCN2 and FGF2 was $5.5 \mathrm{~nm}$. FGF2 promoted not only the proliferation of cultured chondrocytes but also the production of matrix metalloproteinase (MMP)- 9 and -13 by them; however, FGF2 combined with the CT module, but not with fulllength $\mathrm{CCN}$, nullified the enhanced production of both MMPs and chondrocyte proliferation (Nishida et al. 2011b). CCN2 bound to the FGF receptor 1 with a dissociation constant of $362 \mathrm{~nm}$, whereas the CT module did not (Fig. 3); and FGF2- 
stimulated phosphorylation of ERK1/2, p38 MAPK, and cJun N-terminal kinase was decreased by the CT module, but not by full-length CCN2 (Nishida et al. 2011b). These data suggested that the full-length $\mathrm{CCN} 2$ can activate intracellular FGF2 signaling, resulting stimulation of proliferation of chondrocytes and MMP production by chondrocytes. In contrast the cleaved CT module can bind to FGF2 but not to FGFR1 and so cannot activate FGF2 signaling (Nishida et al. 2011b). These findings suggest that CCN2 may regulate the proliferation and matrix degradation of chondrocytes by forming a complex with FGF2 as a novel modulator of FGF2 functions.

By screening a protein array, we also found that $\mathrm{CCN} 2$ could bind to fibroblast growth factor receptors (FGFRs) 2 and 3 (Aoyama et al. 2012). We ascertained that FGFR2 bound to $\mathrm{CCN} 2$ by performing a solid-phase binding assay and SPR. CCN2 enhanced the binding of FGFR2 to FGF2 and FGF4 at least through its TSP-1 module (Fig. 3). CCN2 and FGF2 had a collaborative effect on the phosphorylation of ERK and the differentiation of osteoblastic MC3T3-E1 cells. These results indicate the biological significance of the binding of CCN2 to FGFR2 in bone metabolism (Aoyama et al. 2012).

In addition to the growth factors and their receptors described above, CCN2 can bind to VEGF through TSP-1CT modules (Inoki et al. 2002), to LRP-1 via the TSP-1 module (Segarini et al. 2001; Gao and Brigstock 2003), and to LRP-6, which is a Wnt co-receptor, via the CT module (Mercurio et al. 2004; Fig. 3). Concerning LRP-1, CCN2 was shown to induce tryrosine phosphorylation of its cytoplasmic domain in fibroblasts (Yang et al. 2004). However, in cartilage LRP-1 not only plays a role as a signaling receptor for CCN2 (Kawata et al. 2010) but also is involved in transcytosis of $\mathrm{CCN} 2$ protein in this avascular tissue, which is a protein transport system newly recognized in cartilage (Kawata et al. 2012).

Although its participating binding domain has not yet been identified, CCN2 is also known to interact with tropomyosin-related kinase A (Trk A), which is one of the neurotrophin receptors (Wahab et al. 2005). TrkA is observed in bone-forming cells during fracture healing (Asaumi et al. 2000) and distraction osteogenesis (Aiga et al. 2006) in both of which CCN2 has been shown to play an important role (Nakata et al. 2002; Kadota et al. 2004). CCN2 (Asano et al. 2005) and TrkA (Tsuboi et al. 2001) are also expressed in periodontal ligament cells, suggesting possible interaction therein.

\section{Specific receptors}

By means of a conventional receptor binding assay using iodinated CCN2 and a cross-linking study, a $280-\mathrm{kDa}$ cellsurface molecule was identified on chondrocytes (Nishida et al. 1998), osteoblasts (Nishida et al. 2000), and vascular endothelial cells (Takigawa 2003). However, the structure of this molecule has not been clarified. Although we cannot exclude the possibility that this molecule is identical to one of the already known binding partners, cloning of this molecule is highly expected.
Fig. 7 How CCN2 exhibits such a multifunctionality? CCN2 not only acts through its own receptors but also modifies the actions of various growth factors by binding to them and their receptors. Moreover, it also binds to ECM, which accumulates growth factors around the cells and modifies the actions of these growth factors. The sum of these actions would become the final outcome of the biological action of CCN2. In other words, the various biological actions of CCN2 would depend on the different microenvironments of various types of cells and the state of their differentiation

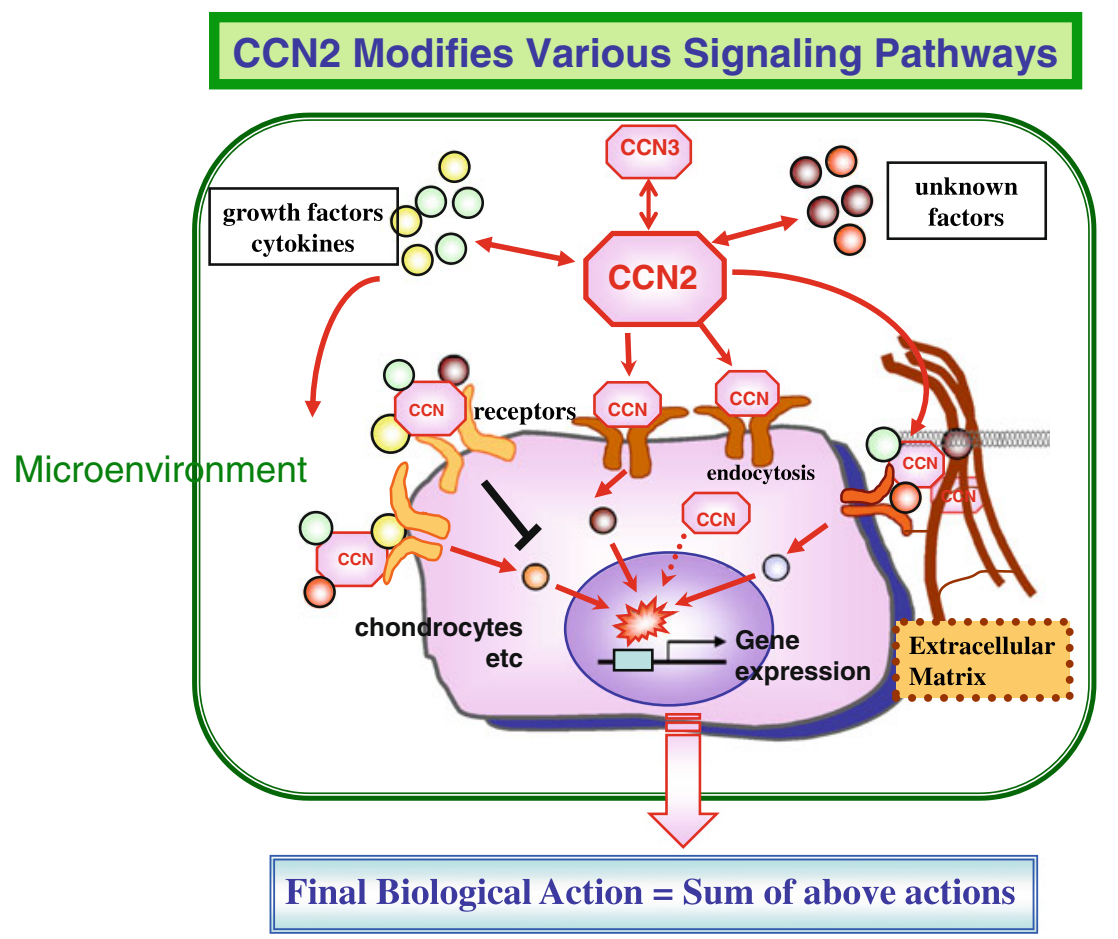

Final Biological Action $=$ Sum of above actions 


\section{CCN2 proteins}

Yeast two-hybrid screening using a cDNA library derived from a chondrocytic cell line, HCS-2/8, identified CCN2 and CCN polypeptides as CCN2-binding proteins (Hoshijima et al. 2012). Direct binding between CCN2 and CCN2 and between $\mathrm{CCN} 2$ and $\mathrm{CCN} 3$ was confirmed by coimmunoprecipitation and SPR, and the calculated dissociation constant $\left(K_{\mathrm{d}}\right)$ was $1.17 \times 10^{-9} \mathrm{M}$ between $\mathrm{CCN} 2$ and CCN2, and $1.95 \times 10^{-9} \mathrm{M}$ between CCN2 and CCN3 (Hoshijima et al. 2012). Endogenous CCN2 and CCN3 interact and co-localize in chondrocytic HCS-2/8 cells, and ectopically overexpressed CCN2 and CCN3 also interact and co-localize in COS7 cells, as determined by direct fluorescence analysis (Hoshijima et al. 2012). CCN2-CCN3 interactions modulate CCN2 activity such as causing enhancement of aggrecan and col2al expression (Hoshijima et al. 2012). Curiously, CCN2 enhances, whereas $\mathrm{CCN} 3$ inhibits, the expression of aggrecan and col2al mRNAs in HCS-2/8 cells (Hoshijima et al. 2012) and proteoglycan synthesis by mouse rib chondrocytes in primary culture (Kawaki et al. 2008a); and the combined treatment with CCN2 and CCN3 abolishes the inhibitory effects of CCN3 (Hoshijima et al. 2012; Kawaki et al. 2008a). An antibody against the VWC domain of CCN2 (11H3) diminishes the binding between $\mathrm{CCN} 2$ and $\mathrm{CCN} 2$, but enhances that between CCN3 and CCN2 (Hoshijima et al. 2012). The results suggest that $\mathrm{CCN} 2$ can form homotypic and heterotypic dimers with $\mathrm{CCN} 2$ and $\mathrm{CCN} 3$, respectively. Enhanced binding between $\mathrm{CCN} 2$ and $\mathrm{CCN} 3$ with the $11 \mathrm{H} 3$ antibody has an enhancing effect on aggrecan expression in chondrocytes, suggesting that the CCN2-CCN3 heterodimer has a positive effect on aggrecan expression in chondrocytes (Hoshijima et al. 2012). We previously reported that CCN2 stimulates the gene expression of CCN2 itself and inhibits that of $\mathrm{CCN} 3$, that $\mathrm{CCN} 3$ inhibits the gene expression of CCN2 and has no effect on $\mathrm{CCN} 3$ expression, and that expression of $\mathrm{CCN} 3$ occurs earlier than that of CCN2 in the process of chondrocyte differentiation toward endochondral ossification (Kawaki et al. 2008a). Therefore, CCN3 suppresses CCN2 expression and chondrocyte differentiation in the early stage of chondrocyte differentiation, but after $\mathrm{CCN} 2$ starts to be expressed, the expressed $\mathrm{CCN} 2$ inhibits $\mathrm{CCN} 3$ expression and stimulates $\mathrm{CCN} 2$ expression, resulting in the promotion of differentiation. In this process, the CCN2-CCN3 heterodimer and then next the CCN2 homodimer would act as chondrocyte differentiation factors in this order.

In summary, CCN2 not only acts through its own receptors but also modifies the actions of various growth factors by binding to them and their receptors (Fig. 7). Moreover, it also binds to ECMs, which accumulate growth factors around the cells and modifies the actions of these growth factors (Fig. 7). The sum of these actions would become the final outcome of biological action of CCN2 (Fig. 7). Moreover, gene expression and activity of CCN2 is also regulated by another member of the $\mathrm{CCN}$ family of proteins, $\mathrm{CCN} 3$ (Fig. 7). In other words, various biological actions of $\mathrm{CCN} 2$ would depend on the different microenvironments of various types of cells as well as on their state of differentiation. Through these various mechanisms, CCN2 would orchestrate various signaling pathways by acting as a signal conductor to promote harmonized skeletal growth and maintenance (Fig. 7).

\section{Future prospects and conclusions}

Evidence thus far indicates that the most important physiological role of CCN2 is the promotion of endochondral ossification. Roles in regeneration, protection of bone and articular cartilage, and angiogenesis are also important ones for CCN2. Continuous search efforts to find other binding partners of CCN2 and to identify the corresponding binding modules in the CCN2 molecule, as well as further investigation of combinational effects, may lead to a better understanding of the molecular mechanisms responsible for the multifunctionality of the CCN2 protein.

Acknowledgments I thank Drs. S. Kubota, T. Hattori, T. Nishida, E. Aoyama, and other faculty members and graduate students who collaborated with us at the Okayama University Graduate School of Medicine, Dentistry, and Pharmaceutical Sciences. This work was supported by the program Grants-in-Aid for Scientific Research (S; No. 19109008) and (B; No. 24390415) and Exploratory Research (No. 23659872) from the Japan Society for the Promotion of Science.

Open Access This article is distributed under the terms of the Creative Commons Attribution License which permits any use, distribution, and reproduction in any medium, provided the original author(s) and the source are credited.

\section{References}

Abreu JG, Ketpura NI, Reversade B, De Robertis EM (2002) Connective-tissue growth factor (CTGF) modulates cell signalling by BMP and TGF-beta. Nat Cell Biol 4:599-604

Aiga A, Asaumi K, Lee YJ, Kadota H, Mitani S, Ozaki T, Takigawa M (2006) Expression of neurotrophins and their receptors tropomyosin-related kinases (Trk) under tension-stress during distraction osteogenesis. Acta Med Okayama 60:267-277

Aigner T, Bertling W, Stoss H, Weseloh G, von der Mark K (1993) Independent expression of fibril-forming collagens I, II, and III in chondrocytes of human osteoarthritic cartilage. J Clin Invest 91:829-837

Aoyama E, Hattori T, Hoshijima M, Araki D, Nishida T, Kubota S, Takigawa M (2009) N-terminal domains of CCN protein 2/connective tissue growth factor bind to aggrecan. Biochem J 420:413-420

Aoyama E, Kubota S, Takigawa M (2012) CCN2/CTGF binds to fibroblast growth factor receptor 2 and modulates its signaling. FEBS Lett 586:4270-4275

Aoyama E, Kubota S, Nishida T, Takigawa M (2013) A novel role of CCN2 in RANK/RANKL/OPG signaling. J Dent Res 92(Special issue A): 1329 
Araki D, Hattori T, Aoyama E, Hoshijima M, Takigawa M (2008) Cartilage growth and differentiation factor CCN2/CTGF binds to matrillin-3, resulting in matrix network formation. J Oral Biosci 50(Suppl): 179

Asano M, Kubota S, Nakanishi T, Nishida T, Yamaai T, Yosimichi G, Ohyama K, Sugimoto T, Murayama Y, Takigawa M (2005) Effect of connective tissue growth factor (CCN2/CTGF) on proliferation and differentiation of mouse periodontal ligament-derived cells. Cell Commun Signal 3:11 (Epub Journal)

Asaumi K, Nakanishi T, Asahara H, Inoue H, Takigawa M (2000) Expression of neurotrophins and their receptors (TRK) during fracture healing. Bone 26:625-633

Brigstock DR (1999) The connective tissue growth factor/cysteine-rich 61/nephroblastoma overexpressed (CCN) family. Endocr Rev 20:189-206

Brigstock DR, Goldshemeding R, Katsube KI, Lam SC, Lau LF, Lyons K, Naus C, Perbal B, Riser B, Takigawa M, Yeger H (2003) Proposal for a unified CCN nomenclature. Mol Pathol 56:127-128

Fujisawa T, Hattori T, Ono M, Uehara J, Kubota S, Kuboki T, Takigawa M (2008) CCN family 2 / connective tissue growth factor (CCN2/CTGF) stimulates proliferation and differentiation of auricular chondrocytes. Osteoarthritis Cartilage 16:787-795

Gao R, Brigstock DR (2003) Low density lipoprotein receptor-related protein (LRP) is a heparin-dependent adhesion receptor for connective tissue growth factor (CTGF) in rat activated hepatic stellate cells. Hepatol Res 27:214-220

Gao R, Brigstock DR (2004) Connective tissue growth factor (CCN2) induces adhesion of rat activated hepatic stellate cells by binding of its C-terminal domain to integrin alpha(v)beta(3) and heparan sulfate proteoglycan. J Biol Chem 279:8848-8855

Hashimoto G, Inoki I, Fujii Y, Aoki T, Ikeda E, Okada Y (2002) Matrix metalloproteinases cleave connective tissue growth factor and reactivate angiogenic activity of vascular endothelial growth factor 165. J Biol Chem 277:36288-36295

Hoshijima M, Hattori T, Inoue M, Araki D, Hanagata H, Miyauchi A, Takigawa M (2006) CT domain of CCN2/CTGF directly interacts with fibronectin and enhances cell adhesion of chondrocytes ?integrin alpha5beta1. FEBS Lett 580:1376-1382

Hoshijima M, Hattori T, Aoyama E, Nishida T, Yamashiro T, Takigawa M (2012) Roles of heterotypic CCN2/CTGF-CCN3/NOV and homotypic CCN2-CCN2 interactions in expression of the differentiated phenotype of chondrocytes. FEBS J 279:3584-3597

Hu JP, Nishishita K, Sakai E, Yoshida H, Kato Y, Tsukuba T, Okamoto K (2008) Berberine inhibits RANKL-induced osteoclast formation and survival through suppressing the NF-kappaB and Akt pathways. Eur J Pharmacol 580:70-79

Inoki I, Shiomi T, Hashimoto G, Enomoto H, Nakamura H, Makino K, Ikeda E, Takata S, Kobayashi K, Okada Y (2002) Connective tissue growth factor binds vascular endothelial growth factor (VEGF) and inhibits VEGF-induced angiogenesis. FASEB J 16:219-221

Itoh S, Hattori T, Tomita N, Aoyama E, Yamashiro T, Takigawa M (2009) CCN2/CTGF has anti-aging effects to protect articular cartilage from age-related degenerative changes. Bone 44:S47

Itoh S, Hattori T, Tomita N, Aoyama E, Yamashiro T, Takigawa M (2011) Cartilage-specific overexpression of CCN2/CTGF protects articular cartilage from age-related osteoarthritis-like changes. J Cell Commun Signal 5:11

Jun JI, Lau LF (2011) Taking aim at the extracellular matrix: CCN proteins as emerging therapeutic targets. Nat Rev Drug Discov 10:945-963

Kadota H, Nakanishi T, Asaumi K, Yamaai T, Nakata E, Mitani S, Aoki K, Aiga A, Inoue H, Takigawa M (2004) Expression of connective tissue growth factor/hypertrophic chondrocytespecific gene product $24(\mathrm{CTGF} / \mathrm{Hcs} 24 / \mathrm{CCN} 2)$ during distraction osteogenesis. J Bone Miner Metab 22:293-302

Kanyama M, Kuboki T, Akiyama K, Nawachi K, Miyauchi FM, Yatani H, Kubota S, Nakanishi T, Takigawa M (2003) Connective tissue growth factor expressed in rat alveolar bone regeneration sites after tooth extraction. Arch Oral Biol 48:723-730

Kawaguchi H (2008) Endochondral ossification signals in cartilage degradation during osteoarthritis progression in experimental mouse models. Mol Cells 25:1-6

Kawaki H, Kubota S, Suzuki A, Lazar N, Yamada T, Matsumura T, Ohgawara T, Maeda T, Perbal B, Lyons KM, Takigawa M (2008a) Cooperative regulation of chondrocyte differentiation by $\mathrm{CCN} 2$ and $\mathrm{CCN} 3$ shown by a comprehensive analysis of the $\mathrm{CCN}$ family proteins in cartilage. J Bone Miner Res 23:1751-1764

Kawaki H, Kubota S, Suzuki A, Yamada T, Matsumura T, Mandai T, Yao M, Maeda T, Lyons KM, Takigawa M (2008b) Functional requirement of $\mathrm{CCN} 2$ for intramembranous bone formation in embryonic mice. Biochem Biophys Res Commun 366:450-456

Kawaki H, Kubota S, Suzuki A, Suzuki M, Kohsaka K, Hoshi K, Fujii T, Lazar N, Ohgawara T, Maeda T, Perbal B, Takano-Yamamoto T, Takigawa M (2011) Differential roles of CCN family proteins during osteoblast differentiation: involvement of Smad and MAPK signaling pathways. Bone 49:975-989

Kawata K, Kubota S, Eguchi T, Moritani NM, Shimo T, Kondo S, Nishida T, Minagi S, Takigawa M (2010) Role of the low-density lipoprotein-1 in regulation of chondrocyte differentiation. J Cell Physiol 222:138-148

Kawata K, Kubota S, Eguchi T, Aoyama E, Moritani NH, Kondo S, Nishida T, Takigawa M (2012) Role of low-density lipoprotein receptor related protein 1 (LRP1) in $\mathrm{CCN} 2 /$ connective tissue growth factor (CTGF) protein transport in chondrocytes. J Cell Sci 125:2965-2972

Kikuchi T, Kubota S, Asaumi K, Kawaki H, Nishida T, Kawata K, Mitani S, Tabata Y, Ozaki T, Takigawa M (2008) Promotion of bone regeneration by $\mathrm{CCN} 2$ incorporated into gelatin hydrogel. Tissue Eng Part A 14:1089-1098

Kubota S, Takigawa M (2007a) CCN family proteins and angiogenesis: from embryo to adulthood. Angiogenesis 10:1-11

Kubota S, Takigawa M (2007b) Role of CCN2/CTGF/Hcs24 in bone growth. Int Rev Cytol 257:1-41

Kubota S, Takigawa M (2011) The role of CCN2 in cartilage and bone development. J Cell Commun Signal 5:209-2017

Lau LF, Lam SC (1999) The CCN family of angiogenic regulators: the integrin connection. Exp Cell Res 248:44-57

Maeda A, Nishida T, Aoyama E, Kubota S, Lyons KM, Kuboki T, Takigawa M (2009) CCN family 2/connective tissue growth factor modulates BMP signaling as a signal conductor, which action regulates the proliferation and differentiation of chondrocytes. $\mathrm{J}$ Biochem 145:207-216

Mercurio S, Latinkic B, Itasaki N, Krumlauf R, Smith JC (2004) Connectivetissue growth factor modulates WNT signalling and interacts with the WNT receptor complex. Development 131:2137-2147

Mori T, Kawara S, Shinozaki M, Hayashi N, Kakinuma T, Igarashi A, Takigawa M, Nakanishi T, Takehara K (1999) Role and interaction of connective tissue growth factor with transforming growth factor- $\beta$ in persistent fibrosis: a mouse fibrosis model. J Cell Physiol 181:153-159

Moussad EE, Brigstock DR (2000) Connective tissue growth factor: what's in a name? Mol Genet Metab 71:276-292

Nakanishi T, Kimura Y, Tamura T, Ichikawa H, Yamaai Y, Sugimoto T, Takigawa M (1997) Cloning of a mRNA preferentially expressed in chondrocytes in differential display PCR from a human chondrocytic cell line that is identical with connective tissue growth factor (CTGF) mRNA. Biochem Biophys Res Commun 234:206-210

Nakanishi T, Nishida T, Shimo T, Kobayashi K, Kubo T, Tamatani T, Tezuka K, Takigawa M (2000) Effects of CTGF/Hcs24, a product of a hypertrophic chondrocyte-specific gene, on the proliferation and differentiation of chondrocytes in culture. Endocrinology $141: 264-273$ 
Nakata E, Nakanishi T, Kawai A, Asaumi K, Yamaai T, Asano M, Nishida T, Mitani S, Inoue H, Takigawa M (2002) Expression of connective tissue growth factor/hypertrophic chondorocytespecific gene product $24(\mathrm{CTGF} / \mathrm{Hcs} 24)$ during fracture healing. Bone 31:441-447

Nishida T, Nakanishi T, Shimo T, Asano M, Hattori T, Tamatani T, Tezuka K, Takigawa M (1998) Demonstration of receptors specific for connective tissue growth factor on a human chondrocytic cell line (HCS-2/8). Biochem Biophys Res Commun 247:905-909

Nishida T, Nakanishi T, Asano M, Shimo T, Takigawa M (2000) Effects of CTGF/Hcs24, a hypertrophic chondrocyte-specific gene product, on the proliferation and differentiation of osteoblastic cells in vitro. J Cell Physiol 184:197-206

Nishida T, Kubota S, Nakanishi T, Kuboki T, Yosimichi G, Kondo S, Takigawa M (2002) CTGF/Hcs24, a hypertrophic chondrocyte specific gene product, stimulates the proliferation and expression of the cartilage phenotype but not hypertrophy or calcification or articular cartilage in culture. J Cell Physiol 192:55-63

Nishida T, Kubota S, Fukunaga T, Kondo S, Yosimichi G, Nakanishi T, Takano-Yamamoto T, Takigawa M (2003) CTGF/Hcs24, hypertrophic chondrocyte-specific gene product, interacts with perlecan in regulating the proliferation and differentiation of chondrocytes. J Cell Physiol 196:265-275

Nishida T, Kubota S, Kojima S, Kuboki T, Nakao K, Kushibiki T, Tabata Y, Takigawa M (2004) Regeneration of defects in articular cartilage in rat knee joints by $\mathrm{CCN} 2$ (connective tissue growth factor). J Bone Miner Res 19:1308-1319

Nishida T, Emura K, Kubota S, Lyons KM, Takigawa M (2011a) CCN family $2 /$ connective tissue growth factor (CCN2/CTGF) promotes osteoclastogenesis via induction of and interaction with dendritic cell-specific transmembrane protein (DC-STAMP). J Bone Miner Res 26:351-363

Nishida T, Kubota S, Aoyama E, Janune D, Maeda A, Takigawa M (2011b) Effect of CCN2 on FGF2-induced proliferation and MMP9 and MMP13 productions by chondrocytes. Endocrinology 152:4232-4241

Ornits DM (2005) FGF signaling in the developing endochondral skeleton. Cytokine Growth Factor Rev 16:205-213

Perbal B, Takigawa M (2005) CCN proteins : a new family of cell growth and differentiation regulators. Imperial College Press, London, pp 1-311

Sato S, Nagaoka T, Hasegawa M, Tamatani T, Nakanishi T, Takigawa M, Takehara K (2000) Serum levels of connective tissue growth factor are elevated in patients with systemic sclerosis: association with the extent of skin sclerosis and the severity of pulmonary fibrosis. J Rheumatol 27:149-154

Segarini PR, Nesbitt JE, Li D, Hays LG, Yates JR 3rd, Carmichael DF (2001) The low density lipoprotein receptor-related protein/alpha2-macroglobulin receptor is a receptor for connective tissue growth factor. J Biol Chem 276:40659-40667

Shimo T, Nakanishi T, Kimura Y, Nishida T, Ishizeki K, Matsumura T, Takigawa M (1998) Inhibition of endogenous expression of connective tissue growth factor by its antisense oligonucleotide and antisense RNA suppresses proliferation and migration of vascular endothelial cells. J Biochem 124:130-140
Shimo T, Nakanishi T, Nishida T, Asano M, Kanyama M, Kuboki T, Tamatani T, Tezuka K, Takemura M, Matsumura T, Takigawa M (1999) Connective tissue growth factor induces the proliferation, migration and tube formation of vascular endothelial cells in vitro and angiogenesis in vivo. J Biochem 126:137-145

Shlopov BV, Gumanovskaya ML, Hasty KA (2000) Autocrine regulation of collagenase 3 (matrix metalloproteinase 13) during osteoarthritis. Arthritis Rheum 43:195-205

Shui C, Riggs BL, Khosla S (2002) The immunosuppressant rapamycin, alone or with transforming growth factor-beta, enhances osteoclast differentiation of RAW264.7 monocytemacrophage cells in the presence of RANK-ligand. Calcif Tissue Int 71:437-446

Takigawa M (2003) CTGF/Hcs24 as a multifunctional growth factor for fibroblasts, chondrocytes, and vascular endothelial cells. Drug News Perspect 16(11-21):2003

Takigawa M, Nakanishi T, Kubota S, Nishida T (2003) The role of CTGF/Hcs24/ecogenin in skeletal growth control. J Cell Physiol 194:256-266

Tomita N, Hattori T, Ito S, Aoyama, E, Yao M, Yamashiro T and Takigawa M (2013) Cartilage-specific overexpression of CCN family member $2 /$ connective tissue growth factor (CCN2/CTGF) stimulates insulin-like growth factor expression and bone growth. Plos One 8:e59226

Tsuboi Y, Nakanishi T, Takano-Yamamoto T, Miyamoto M, Yamashiro T, Takigawa M (2001) Mitogenetic effect of neurotrophins on periodontal ligament cell line. J Dent Res 80:881-886

Vial C, Gutiérrez J, Santander C, Cabrera D, Brandan E (2011) Decorin interacts with connective tissue growth factor (CTGF)/CCN2 by LRR12 inhibiting its biological activity. J Biol Chem 286:2424224252

Wahab NA, Weston BS, Mason RM (2005) Connective tissue growth factor CCN2 interacts with and activates the tyrosine kinase receptor TrkA. J Am Soc Nephrol 16:340-351

Yamashiro T, Fukunaga T, Kobashi N, Kamioka H, Nakanishi T, Takigawa M, Takano-Yamamoto T (2001) Mechanical stimulation induces CTGF expression in rat osteocytes. J Dent Res $80: 461-465$

Yang M, Huang H, Li J, Li D, Wang H (2004) Tyrosine phosphorylation of the LDL receptor-related protein (LRP) and activation of the ERK pathway are required for connective tissue growth factor to potentiate myofibroblast differentiation. FASEB J 18:19201921

Yoon BS, Lyons KM (2004) Multiple functions of BMPs in chondrogenesis. J Cell Biochem 93:93-103

Yosimichi G, Nakanishi T, Nishida T, Hattori T, Takano-Yamamoto T, Takigawa M (2001) CTGF/Hcs24 induces chondrocyte differentiation through a p38 mitogen-activated protein kinase (p38MAPK), and proliferation through p44/42 MAPK/ exracellular-signal regulated kinase (ERK). Eur J Biochem 268:1-9

Yosimichi G, Kubota S, Nishida T, Kondo S, Yanagita T, Nakao K, Takano-Yamamoto T, Takigawa M (2006) Roles of PKC, PI3K and JNK in multiple transduction of CCN2/CTGF signals in chondrocytes. Bone 38:853-863 